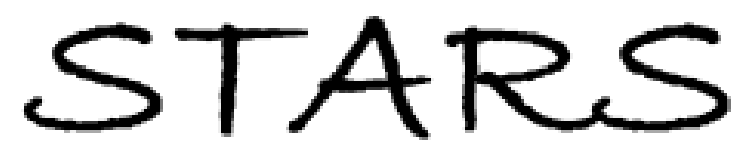

University of Central Florida

STARS

$1-1-2004$

\title{
Low-loss wavelength-multiplexed optical scanners using volume Bragg gratings for transmit-receive lasercom systems
}

Zahid Yaqoob

University of Central Florida

Nabeel A. Riza

University of Central Florida

Find similar works at: https://stars.library.ucf.edu/facultybib2000 University of Central Florida Libraries http://library.ucf.edu

This Article is brought to you for free and open access by the Faculty Bibliography at STARS. It has been accepted for inclusion in Faculty Bibliography 2000 s by an authorized administrator of STARS. For more information, please contact STARS@ucf.edu.

\section{Recommended Citation}

Yaqoob, Zahid and Riza, Nabeel A., "Low-loss wavelength-multiplexed optical scanners using volume Bragg gratings for transmit-receive lasercom systems" (2004). Faculty Bibliography 2000s. 4906.

https://stars.library.ucf.edu/facultybib2000/4906

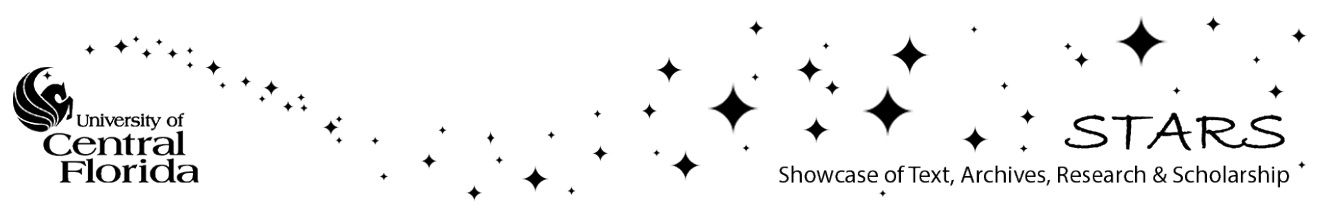




\section{Low-loss wavelength-multiplexed optical scanners using volume Bragg gratings for transmit-receive lasercom systems}

\section{Zahid Yaqoob, Nabeel Agha Riza}

Zahid Yaqoob, Nabeel Agha Riza, "Low-loss wavelength-multiplexed optical scanners using volume Bragg gratings for transmit-receive lasercom systems," Proc. SPIE 5160, Free-Space Laser Communication and Active Laser Illumination III, (27 January 2004); doi: 10.1117/12.504378

SPIE Event: Optical Science and Technology, SPIE's 48th Annual Meeting, 2003, San Diego, California, United States 


\title{
Low-Loss Wavelength-Multiplexed Optical Scanners using Volume Bragg Gratings for Transmit-Receive Lasercom Systems
}

\author{
Zahid Yaqoob and Nabeel A. Riza \\ Photonic Information Processing Systems (PIPS) Laboratory \\ The School of Optics/Center for Research and Education in Optics and Lasers (CREOL) \\ University of Central Florida, 4000 Central Florida Blvd., Orlando, FL 32816-2700 \\ Tel: 407-823-6829; Fax: 407-823-6880 \\ E-mail: nriza@mail.ucf.edu
}

\begin{abstract}
Low-loss no moving parts free-space wavelength-multiplexed optical scanner (W-MOS) modules for transmit-receive lasercom systems are proposed and experimentally demonstrated. The proposed scanners are realized using volume Bragg gratings stored in dichromated gelatin (DCG) coupled with high-speed wavelength selection such as by a fast tunable laser. The potential speed of these scanners is in the Gigahertz range using present-day state-of-the-art nanosecond tuning speed lasers. A 940-lines $/ \mathrm{mm}$ volume Bragg grating stored in dichromated gelatin is used to demonstrate the scanners. Angular dispersion and diffraction efficiency of the volume Bragg grating used for demonstration are studied versus wavelength and angle of incidence to determine the free-space W-MOS angular scan and insertion loss, respectively. Experimental results show that a tunable bandwidth of $80 \mathrm{~nm}$, centered at $1560 \mathrm{~nm}$, delivers an angular scan of $6.25^{\circ}$. The study also indicates that an in-line scanner design realized using two similar Bragg gratings in DCG delivers $13.42^{\circ}$ angular scan, which is more than double the angular scan available from the free-space W-MOS using single volume Bragg grating.
\end{abstract}

Keywords: Optical scanners, Free-space Lasercom, Bragg diffraction gratings, Wavelength-multiplexing

\section{INTRODUCTION}

Free-space optical communication systems due to their large information capacity are emerging as an attractive choice for alleviating data communication bottlenecks at different levels of the information highway. ${ }^{1,2}$ The narrow beam width of the laser is one of the major advantages of free-space optical communications as it allows sending information with high directivity (low side lobes) and security, which in-turn makes the system efficient in power consumption. The high directivity of a laser beam, however, brings forward serious challenges related to beam-pointing technology in free-space optical communication systems. The problem is compounded by the fact that the amplitude of vibrations in moving platforms is much bigger than the laser beam size. ${ }^{3}$ Thus beam pointing and tracking control system with fast and agile fine beam pointing (e.g., $\pm 5 \mu \mathrm{rad})^{4}$ capability needs to be an integral part of a lasercom subsystem to reduce moving platform vibration errors for reduced data bit error rates. Specifically, there is a need to realize a large aperture (e.g., 10 $\mathrm{cm}$ diameter) scanner that also has low power consumption (e.g., $\mathrm{mWs}$ ) and is rapidly (e.g., a few $\mu \mathrm{s}$ ) reconfigurable. Hence there exists a size versus speed dilemma if moving parts_optics is deployed for beam pointing. In this paper, we present scanners using volume Bragg gratings stored in dichromated gelatin that promise low-loss, high-speed, and high resolution one dimensional wide angular scans.

In 1970's, it was realized that laser wavelength changes combined with wavelength sensitive optics could be used for laser beam steering. ${ }^{5,6}$ Independently, we have a proposed, analyzed, and demonstrated how extremely fast_wavelength selection combined with present day planar dispersive elements (e.g., diffraction gratings) can be used to realize onedimensional (1-D) beam scanner, known as the free-space wavelength-multiplexed optical scanner (W-MOS). ${ }^{7}$ Freespace W-MOS simultaneously provides fast speed, large apertures, sub-degree scan resolution, 1-D wide angular scans, and simple control. Experimental demonstration of a free-space W-MOS using blazed reflection grating (600-lines $/ \mathrm{mm})$ 
shows high resolution $(\leq 1 \mathrm{mrad}$ beam divergence for a $1.875 \mathrm{~cm}$ diffraction limited aperture diameter) and large angular scans $\left(\sim 13^{\circ}\right){ }^{8}$ The study indicates that the angular scan range of the scanner can be increased by choosing suitable angle of incidence on the diffraction grating. However, failing to satisfy the Littrow configuration significantly degrades the diffraction efficiency of a blazed grating versus wavelength, hence causing higher scanner insertion loss. Because transmissive surface relief sinusoidal phase gratings cannot deliver high diffraction efficiency in the infrared region $(1550 \mathrm{~nm})$, the alternative would be to use volume Bragg gratings that guarantee clear scanner aperture as well as symmetric design for effective scanner operation. Recently, we proposed and showed, to our knowledge for the first time, how robust high efficiency narrowband tilted volume Bragg gratings in photothermorefractive glass ${ }^{9}, 10$ combined with high-speed wavelength selection can be used to realize the desired fast beam switching large aperture twodimensional scanner. ${ }^{11}$ In this paper, we will present and discuss in detail the functionality of volume Bragg gratings stored in dichromated gelatin (also known as Dickson gratings ${ }^{12}$ ) for use in free-space W-MOS capable of onedimensional beam scanning. Grating design parameters such as loss and diffraction efficiency versus wavelength will be discussed. Based on diffraction efficiency measurements, grating structure thickness and the index of modulation will be estimated. Dickson grating-based free-space W-MOS modules will be presented and scanner parameters such as insertion loss and angular scan versus wavelength of the tunable source will be discussed.

\section{VOLUME PHASE GRATINGS IN DICHROMATED GELATIN}

Volume phase gratings recorded interferometrically in dichromated gelatin (DCG), also known as Dickson gratings, offer very high index of modulation that makes it possible to record volume phase gratings with very small thickness $(<$ $20 \mu \mathrm{m})$ yet able to offer extremely high diffraction efficiency $(>95 \%)$. Furthermore, the small thickness of the volume phase gratings in Dickson gratings makes them less selective in $\lambda$, i.e., almost flat response over the desired wavelength band. The construction of a Dickson grating is shown in Fig. 1..$^{\sharp}$ A thin layer of DCG $(<20 \mu \mathrm{m}$ thick $)$ is deposited on a glass plate [see the glass plate on right hand side in Fig. 1 (b)]. When the volume phase grating is recorded and fixed, matching index glue is applied on the DCG layer before second glass plate [shown on the left hand side in Fig. 1(b)] is placed on it and sealed with a telecom-grade epoxy. Since the bulk index of refraction of DCG is 1.3 and there is no index matching glue between one of the two DCG-glass interfaces, Fresnel reflection loss in the range of $0.04-0.14 \mathrm{~dB}$ is be observed depending upon the index of the glass plate between $1.50-1.70$. The volume phase grating designed for telecom has $940-$ lines $/ \mathrm{mm}$ spatial frequency that corresponds to a grating period $L=1.0638 \mu \mathrm{m}$. At Bragg wavelength $\lambda_{\mathrm{B}}=1550 \mathrm{~nm}$, the Bragg angle in air is $\theta_{\mathrm{B}, \mathrm{air}}=46.76^{\circ}$.

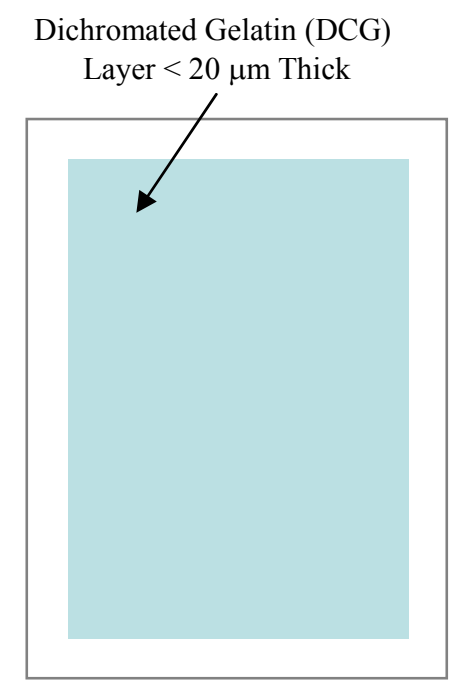

(a)

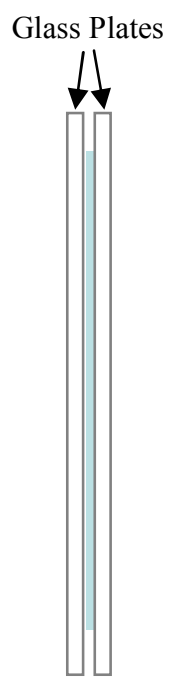

(b)

Figure 1: Schematic of a volume Bragg grating in dichromated gelatin. (a) Front View, (b) Side View.

\footnotetext{
¥ Wasatch Photonics, CA http://www.wasatchphotonics.com
} 
To study the diffraction efficiency of a Dickson grating, collimated beam from a broadband source with $1 / \mathrm{e}^{2}$ beam size of $\sim 1 \mathrm{~mm}$ is incident on the grating mounted on a rotational stage to adjust the angle of incidence (see Fig. 2). The angle of incidence is set to $\theta_{\mathrm{inc}}=\theta_{\mathrm{B}, \text { air }}=46.76^{\circ}$ to satisfy the Bragg condition at $\lambda_{\mathrm{B}}=1550 \mathrm{~nm}$. An optical spectrum analyzer is used to measure the optical power in the incident and undiffracted (zeroth order) beams to determine loss (due to absorption or scattering) and optical power diffracted into the first order to estimate absolute as well as relative diffraction efficiencies of the Dickson grating versus wavelength.

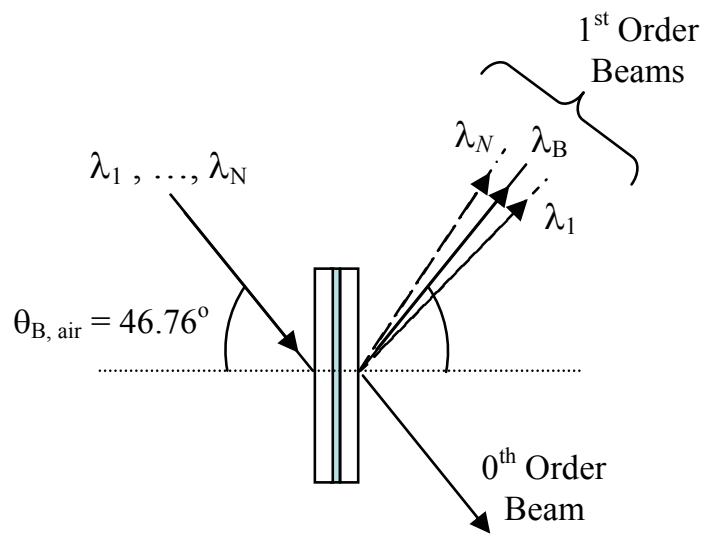

Figure 2: Experimental setup to study loss as well as relative and absolute diffraction efficiencies of a Dickson grating; $\lambda_{\mathrm{B}}=1550 \mathrm{~nm}$.

Figures 3 (a) - (c) show the loss, relative diffraction efficiency, and absolute diffraction efficiency, respectively, versus wavelength of the broadband source in $70 \mathrm{~nm}$ band around $\lambda_{\mathrm{B}}=1550 \mathrm{~nm}$. The study shows that the loss $(\mathrm{dB})$ varies from $0.4 \mathrm{~dB}$ to $0.25 \mathrm{~dB}$ as the wavelength is changed from $1520 \mathrm{~nm}$ to $1590 \mathrm{~nm}$. The average loss over the $70 \mathrm{~nm}$ tunable band is $0.298 \mathrm{~dB}$. The maximum value of the relative diffraction efficiency which is defined as the ratio of $P_{\text {Diff,ON-Bragg }}$ to $\mathrm{P}_{\mathrm{DC} \text {,OFF-Bragg }}$ is measured as $99.75 \%$, where $\mathrm{P}_{\text {Diff,ON-Bragg }}$ is the optical power in the first order diffracted beam at Bragg $\left(\lambda_{\mathrm{B}}\right.$ $=1550 \mathrm{~nm})$ and $\mathrm{P}_{\mathrm{DC}, \text { OFF-Bragg }}$ is the optical power in the undiffracted DC beam when Bragg condition is not satisfied $\left(\theta_{\text {inc,air }} \neq \theta_{\mathrm{B}, \text { air }}\right)$. Figure 3 (b) shows that the relative diffraction efficiency does not drop sharply with the wavelength and is symmetric about $\lambda_{\mathrm{B}}=1550 \mathrm{~nm}$.

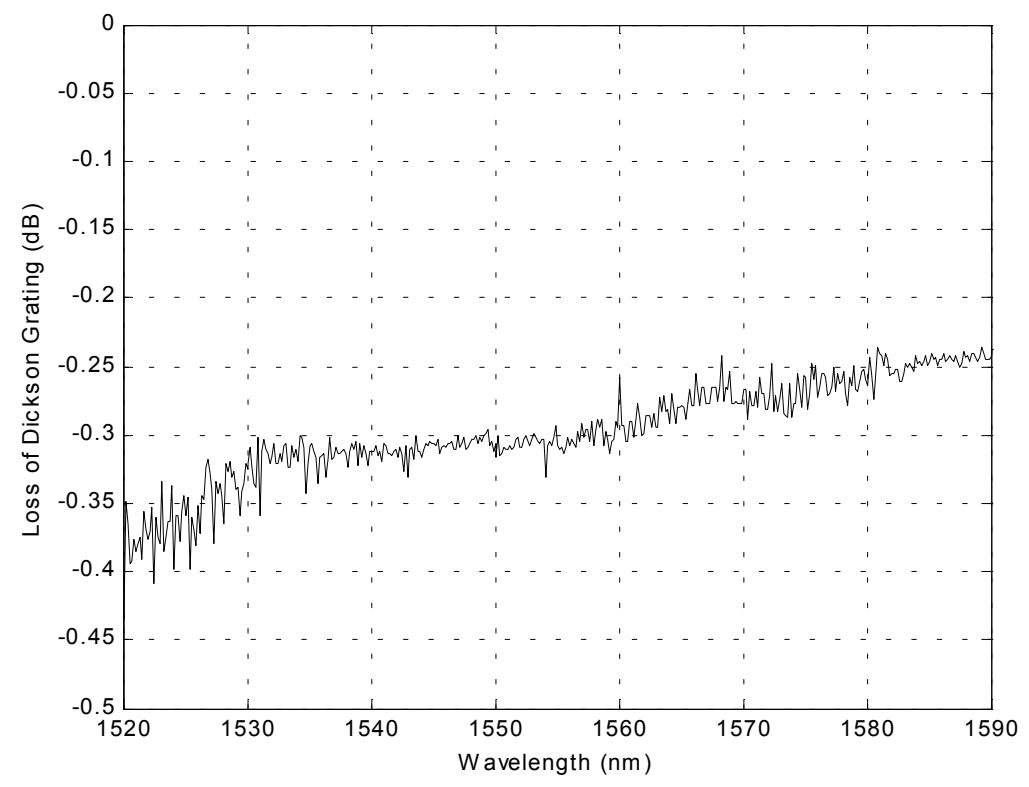

(a) 
Similarly, the study of the absolute diffraction efficiency defined as the ratio of $\mathrm{P}_{\text {Diff,ON-Bragg }}$ to $\mathrm{P}_{\text {inc }}$ shows that the maximum absolute diffraction efficiency is $\sim 93.0 \%$, where $\mathrm{P}_{\text {inc }}$ is the optical power in the incident beam [see Fig. 3 (c)].

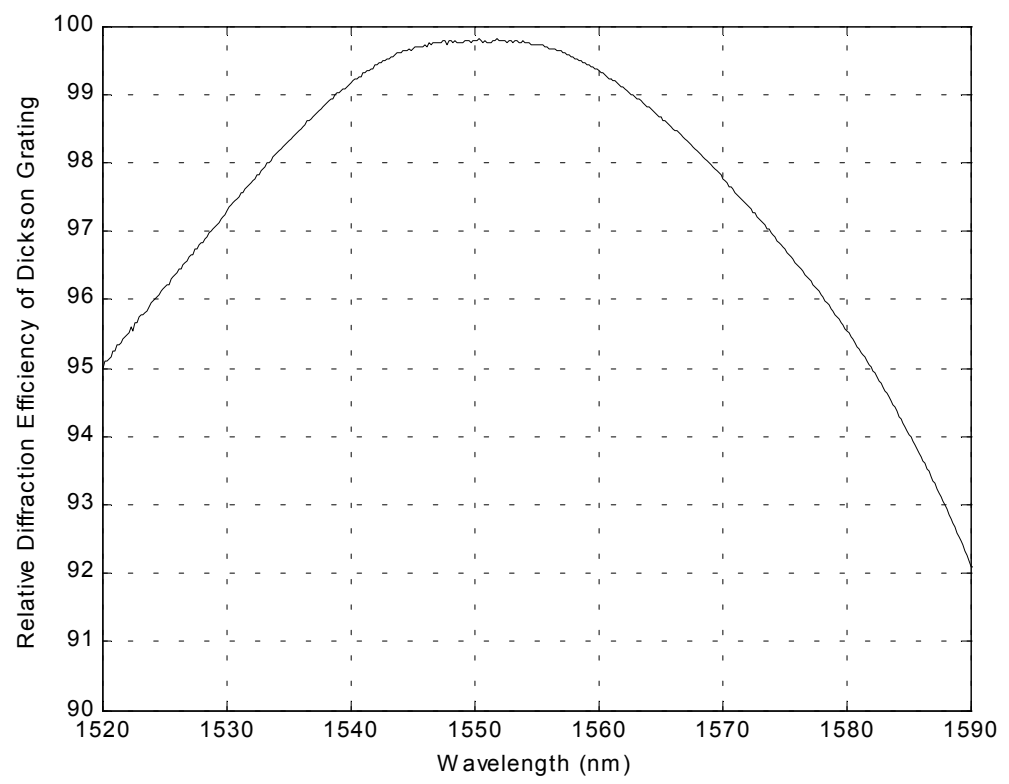

(b)

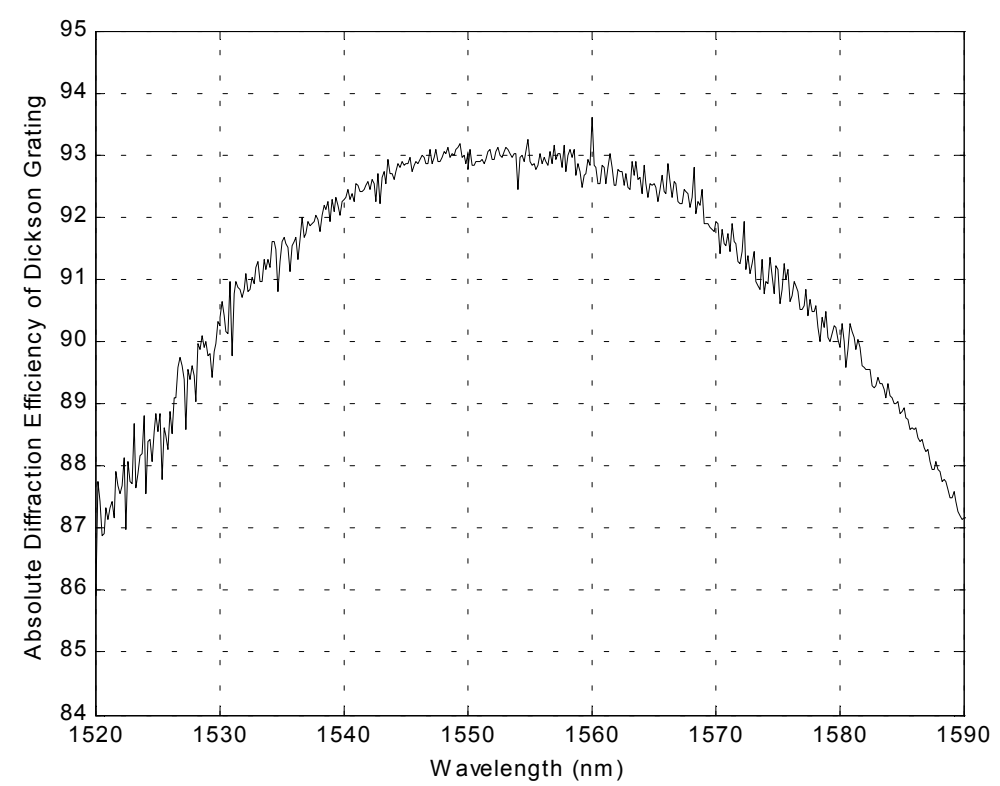

(c)

Figure 3: shows measured (a) loss, (b) relative diffraction efficiency, and (c) absolute diffraction efficiency of the Dickson grating under study versus wavelength; $\lambda_{\mathrm{B}}=1550 \mathrm{~nm}$.

Note that the absolute diffraction efficiency is not symmetric about $\lambda_{B}=1550 \mathrm{~nm}$ because the loss (dB) is not uniform over the wavelength band under consideration. The experimental study also indicates that the absolute diffraction efficiency remains $>87 \%$ over the $70 \mathrm{~nm}$ wavelength band. 
To estimate the thickness $d$ of the DCG layer and the index of modulation $\Delta n$ of the recorded phase grating, the dephasing parameter $\xi$ in the expression for diffraction efficiency $\eta$ given by ${ }^{13}$

$$
\eta=\frac{\gamma^{2}}{\gamma^{2}+\xi^{2}} \sin ^{2}\left[\sqrt{\gamma^{2}+\xi^{2}}\right]
$$

is determined for a known value of $\eta$ at an off-Bragg wavelength $\lambda=\lambda_{B} \pm \Delta \lambda$. Note that $\gamma$ is the coupling parameter given by ${ }^{13}$

$$
\gamma(\lambda)=\frac{\pi \Delta n d}{\lambda \cos \theta_{\text {inc, g }}},
$$

where $\theta_{\text {inc,g }}$ is the angle of incidence inside the DCG layer. Since the maximum relative diffraction efficiency $\eta_{\mathrm{B}}=$ 0.9975 [see Fig. 3(b)], the corresponding value of coupling parameter comes out to be $\gamma_{\mathrm{B}}=1.5208$. The thickness $d$ of the DCG is estimated in an iterative fashion as follows. First of all, the preliminary value of dephasing parameter $\xi$ is calculated from Eq. (1) as 0.349 (assuming that $\gamma=\gamma_{\mathrm{B}}$ at $\lambda=1520 \mathrm{~nm}$ ). Next, the thickness $d$ of the DCG is estimated from the following relation ${ }^{13}$

$$
\frac{\Delta \lambda}{\lambda}=-\frac{\xi L}{\pi d \tan \theta_{\mathrm{inc,g}}},
$$

where $\Delta \lambda=-30 \mathrm{~nm}, L=1.0638 \mu \mathrm{m}$, and $\theta_{\text {inc,g }}=35.969^{\circ}$. The estimated value of thickness $d$ of the DCG layer comes out to be $8.251 \mu \mathrm{m}$. The estimated value of $d$ is then used to determine the index of modulation $\Delta n$ using Eq. (2). By substituting the values of $\gamma_{\mathrm{B}}=1.5208, \lambda_{\mathrm{B}}=1550 \mathrm{~nm}, \theta_{\text {inc, } \mathrm{g}}=35.969^{\circ}$, and $d=8.251 \mu \mathrm{m}$ in Eq. (2), the index of modulation is estimated as $\Delta n=73.6 \times 10^{-3}$. Next, the value of $\gamma$ is determined at $\lambda=1520 \mathrm{~nm}$ using the same relation [Eq. (3)]. Iteratively, the new value of $\gamma$ is used to estimate again values of $\xi, d, \Delta n$, and $\gamma$. Note that the procedure converges very fast (e.g., in 2 to 3 iterations). The final values of $d$ and $\Delta n$ are estimated as $8.393 \mu \mathrm{m}$ and $72.4 \times 10^{-3}$, respectively. Note that this value of $\Delta n$ is almost 72 times the maximum index of modulation possible in photothermorefractive glass.

\section{FREE-SPACE WAVELENGTH-MULTIPLEXED OPTICAL SCANNER USING DICKSON GRATINGS}

As discussed in the earlier section, owing to very high index of modulation $\left(\sim 72.4 \times 10^{-3}\right)$ possible in dichromated gelatin (DCG), volume phase gratings with extremely high diffraction efficiency $(>95 \%)$ can be recorded in very small thickness of the DCG layer. Furthermore, the small thickness of the DCG layer in Dickson gratings makes them less selective in wavelength. Hence, the two properties, i.e., high diffraction efficiency and low wavelength selectivity make Dickson gratings suitable for applications like free-space W-MOS requiring low-loss broadband operations. The scanner insertion loss of free-space W-MOS defined as $10 \times \log \left(\eta_{\text {abs }}\right)$, where $\eta_{\text {abs }}$ is the absolute diffraction efficiency [already discussed and shown in Fig. 3(c)], is shown in Fig. 4. The minimum value of scanner insertion loss is 0.315 at $\lambda_{\mathrm{B}}=1550$ $\mathrm{nm}$. The average scanner insertion loss over the $70 \mathrm{~nm}$ tunable band is $0.4 \mathrm{~dB}$. Because there is at least one dichromated gelatin layer-glass interface that has an index mismatch, Fresnel reflection loss in the range [0.4 - 0.14] dB is expected for glass plate index of refraction in the range [1.5 - 1.7], respectively.

To study the angular scan available from the free-space W-MOS using volume Bragg grating in dichromated gelatin, a setup similar to that shown in Fig. 2. was built in the laboratory. Collimated beam from a fiber-coupled tunable laser source (with $1 / \mathrm{e}^{2}$ beam size of $\sim 0.6 \mathrm{~mm}$ ) is incident on the grating mounted on a rotational stage to adjust the angle of incidence (see Fig. 2). The Bragg angle is set to $\theta_{\mathrm{B}, \text { air }}=46.76^{\circ}$ to satisfy the Bragg condition at $\lambda_{\mathrm{B}}=1550 \mathrm{~nm}$. An infrared camera is used to observe the scanning first diffraction order when the wavelength of the tunable laser is tuned over the $80 \mathrm{~nm}$ wavelength band centered at $1560 \mathrm{~nm}$. The angular deflection is measured by tracking the scanning first diffraction order with the help of an iris mounted on an aluminum C-channel with one end attached to a rotational stage having the same axis of rotation as that for the Bragg grating. A total angular scan of $6.25^{\circ}$ was measured by the tuning the wavelength of the source from $1520 \mathrm{~nm}$ to $1600 \mathrm{~nm}$. Furthermore, the angular scan available over the $80 \mathrm{~nm}$ tunable band is estimated using the grating equation ${ }^{14}$ 


$$
\theta_{\text {out air }}=\sin ^{-1}\left\{\frac{\lambda}{L}-\sin \theta_{\text {inc, air }}\right\},
$$

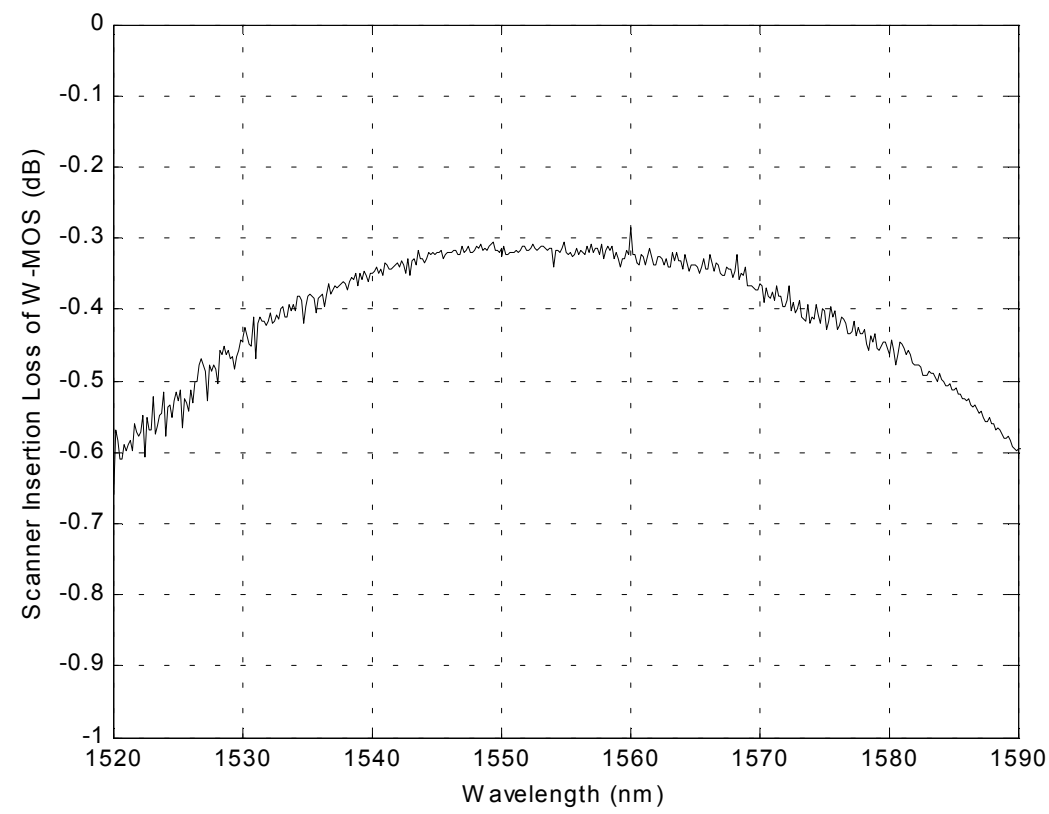

Figure 4: Scanner insertion loss of free-space wavelength-multiplexed optical scanner versus wavelength; $\lambda_{B}=1550 \mathrm{~nm}$.

Figure 5 shows both experimental and theoretical angular scan (in degrees) versus wavelength of the tunable source. The plot indicates that the measured angular scan is almost consistent with the estimated scan of $6.39^{\circ}$ as the source is tuned over the $80 \mathrm{~nm}$ tunable bandwidth around $1560 \mathrm{~nm}$.

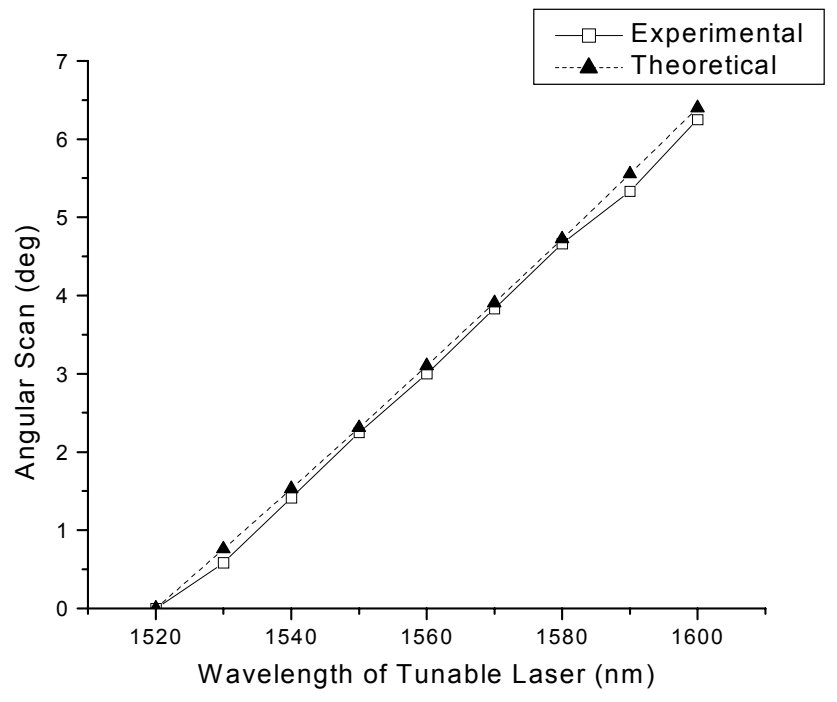

Figure 5: Angular scan available from a free-space W-MOS using a single Dickson grating versus wavelength. 
Equation (4) suggests that an angular scan of $9.48^{\circ}$ is possible if the wavelength of the source is tuned over $120 \mathrm{~nm}$ bandwidth centered at $\lambda_{\mathrm{B}}=1550 \mathrm{~nm}\left(\theta_{\mathrm{inc}}=\theta_{\mathrm{B}, 1550}=46.76^{\circ}\right)$. However, the measured relative diffraction efficiency of the Dickson grating (Bragg set at $1550 \mathrm{~nm}$ ) drops to $\sim 80 \%$ around the edges of the tunable band [see Fig. 6 (a)]. It is important to note that angular scans of $4.53^{\circ}$ and $4.95^{\circ}$ are possible when the grating is Bragg set at $\lambda_{\mathrm{B}}=1520 \mathrm{~nm}\left(\theta_{\text {inc }}=\right.$ $\left.\theta_{\mathrm{B}, 1520}=45.59^{\circ}\right)$ and $1580 \mathrm{~nm}\left(\theta_{\mathrm{inc}}=\theta_{\mathrm{B}, 1580}=47.95^{\circ}\right)$ and the wavelength of the source is tuned from $\lambda=1475-1535$ $\mathrm{nm}$ and $1565-1625 \mathrm{~nm}$, respectively. Moreover, the sum of the two tunable bands and the angular scan ranges are 120 $\mathrm{nm}$ and $9.48^{\circ}$, respectively (same as in the previous case). The relative diffraction efficiency of the Dickson grating for the two cases, i.e., $\lambda_{\mathrm{B}}=1520 \mathrm{~nm}\left(\theta_{\text {inc }}=45.59^{\circ}\right)$ and $1580 \mathrm{~nm}\left(\theta_{\text {inc }}=47.95^{\circ}\right)$ was also measured versus wavelength and the results are shown in Figs. 6(b) and (c). The two plots clearly indicate that the overall relative diffraction efficiency of the Dickson grating is better than the case when the grating is Bragg set at $\lambda_{B}=1550 \mathrm{~nm}$ and the wavelength of the source is tuned over $120 \mathrm{~nm}$ [see Fig. 6(a)]. It can therefore be concluded that it is better to choose smaller distributed tunable bands rather than a single large tunable band to achieve overall higher diffraction efficiency, which indicates lower average scanner insertion but still the same angular scan range. However, it will be required to efficiently adjust the appropriate angle of incidence for each corresponding tunable band.

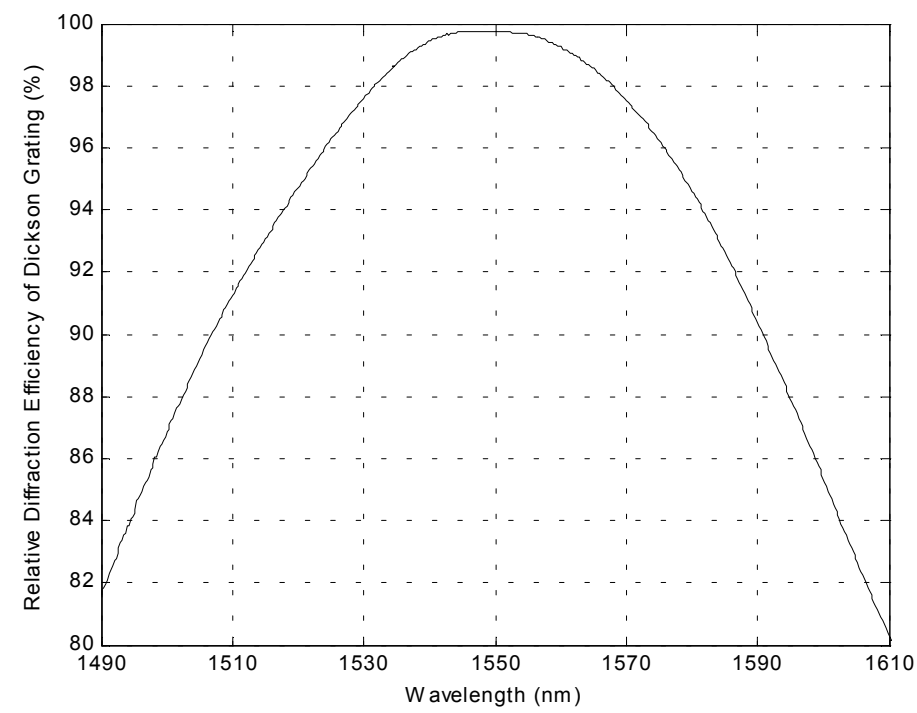

(a)

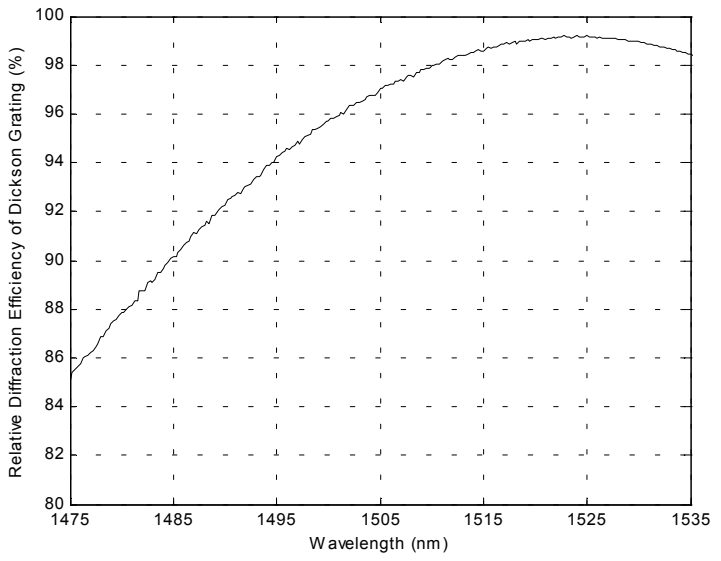

(b)

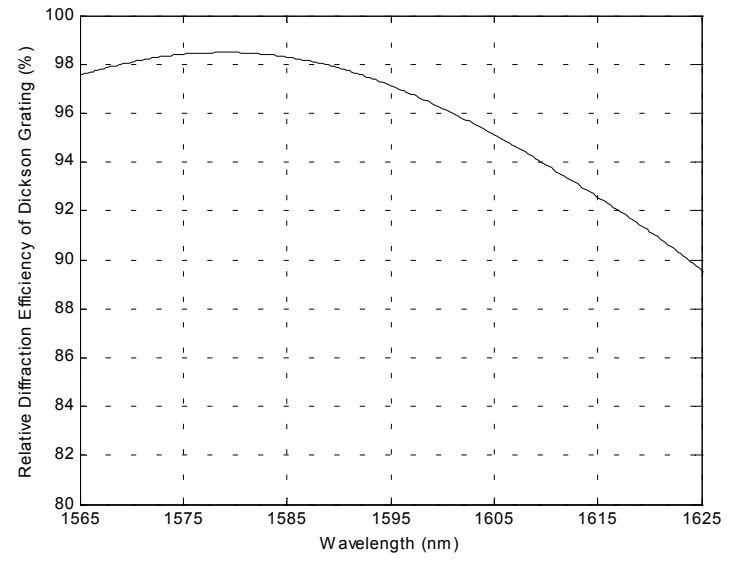

(c)

Figure 6: Relative diffraction efficiency of Dickson grating for (a) $\lambda_{\mathrm{B}}=1550 \mathrm{~nm} ; \theta_{\text {inc }}=46.76^{\circ} ; \lambda=1490-1610 \mathrm{~nm}$, (b) $\lambda_{\mathrm{B}}=1520 \mathrm{~nm}$; $\theta_{\text {inc }}=45.59^{\circ} ; \lambda=1475-1535 \mathrm{~nm}$, and (c) $\lambda_{\mathrm{B}}=1580 \mathrm{~nm} ; \theta_{\text {inc }}=47.95^{\circ} ; \lambda=1565-1625 \mathrm{~nm}$. 
Figure 7 shows the schematic of a free-space W-MOS that uses a fast ( $\mu$ s speed) $1 \times 2$ fiber-coupled broadband optical switch to adjust the angle of incidence on the Dickson grating. The two output fibers of the optical switch end up in an assembly that keeps the polished ends of the single mode fibers (SMFs) at one focal length distance from an achromatic lens. The two SMFs are parallel with each other such that one of the fibers is aligned with the principal axis of the lens whereas the other is shifted by $x^{\prime}$ (see Fig. 7). The elevation angles and $1 / \mathrm{e}^{2}$ beam diameter (BD) of the collimated beams after the lens are given by

$$
\theta^{\prime}=-\tan ^{-1}\left(\frac{x^{\prime}}{f}\right)
$$

and

$$
1 / \mathrm{e}^{2} \mathrm{BD}=\frac{2 \lambda f \cos \theta^{\prime}}{\pi w_{\mathrm{inc}}},
$$

respectively, where $f$ is focal length of the collimating lens and $w_{\text {inc }}$ is the beam waist (half of the mode field diameter) of the Gaussian beam coming out of the SMFs. For instance, using $f=75 \mathrm{~mm}$ focal length lens, $x^{\prime}=3.09 \mathrm{~mm}$ will yield $\theta^{\prime}=$ $2.359^{\circ}$ (the difference in Bragg angles for $\lambda_{\mathrm{B}}=1520$ and $1580 \mathrm{~nm}$ ). Therefore, collimated beam from port 2 of the optical switch will be incident on the Dickson grating at $\theta_{\text {inc }}=47.953^{\circ}$ (the Bragg angle for $\lambda_{\mathrm{B}}=1580 \mathrm{~nm}$ ), whereas the collimated beam from port 1 of the optical switch will be incident on the Dickson grating at $\theta_{\text {inc }}=45.594^{\circ}$ (the Bragg angle for $\lambda_{\mathrm{B}}=1520 \mathrm{~nm}$ ). Hence, from operational point of view, the optical switch will be set to route the input light to port 1 (that will set $\theta_{\text {inc }}=45.594^{\circ}$ on the grating) and the wavelength of the laser will be tuned from $1475 \mathrm{~nm}$ to 1535 $\mathrm{nm}$. Next, the optical switch will be set to route the input light to port 2 (that will set $\theta_{\text {inc }}=47.953^{\circ}$ ) and the wavelength of the laser will be tuned from $1565 \mathrm{~nm}$ to $1625 \mathrm{~nm}$ to get the rest of the angular scan with high overall throughput. Note that the idea can be extended by increasing the number of output ports of the optical switch (such as to three or more).

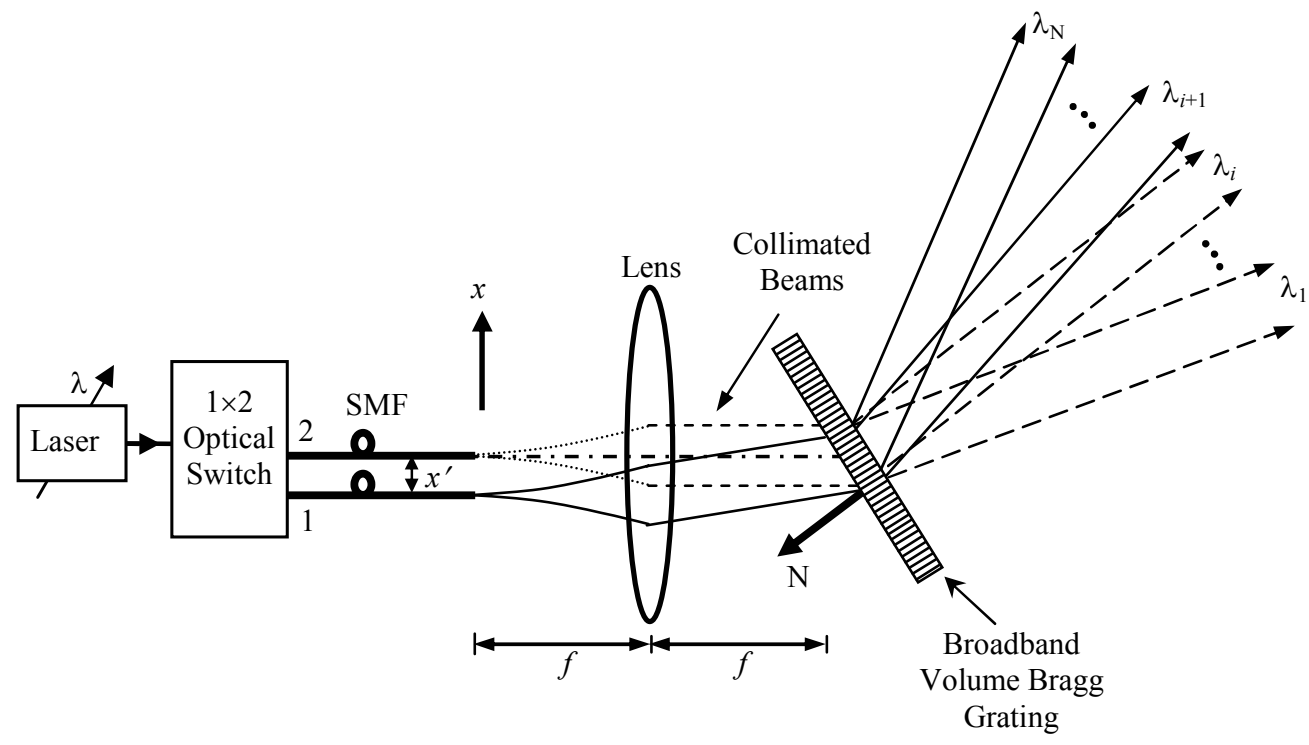

Figure 7: Schematic of a smart free-space W-MOS with incidence angle setting capability for very high overall throughput. N: Grating normal; SMF: Single mode fiber; $f$ : Focal length of the collimating lens.

\section{IN-LINE DESIGN OF A FREE-SPACE WAVELENGTH-MULTIPLEXED OPTICAL SCANNER WITH WIDE ANGULAR SCAN RANGE}

As discussed earlier, because of the high spatial frequency (940 lines $/ \mathrm{mm}$ ) in Dickson gratings, the Bragg angle is significantly large $\left(\theta_{\mathrm{B}}=46.76^{\circ}\right.$ at $\left.\lambda_{\mathrm{B}}=1550 \mathrm{~nm}\right)$ that makes the first order beam to diffract almost in an orthogonal 
direction to the incident beam. A total angular scan of $6.25^{\circ}$ has also been demonstrated by the tuning the wavelength of the incident beam from $1520 \mathrm{~nm}$ to $1600 \mathrm{~nm}$. At this point, the motivation is to design an inline free-space W-MOS that can also deliver increased total angular scan range (using the same tunable bandwidth of the laser source). Figure 8 shows two similar Dickson gratings arranged in a symmetrical fashion such that the angle between the planes of the two gratings is $2 \theta_{\mathrm{B}}$, where $\theta_{\mathrm{B}}$ is the Bragg incidence angle corresponding to the Bragg wavelength $\lambda_{\mathrm{B}}$. Because $\theta_{\text {inc, } 1}=\theta_{\mathrm{B},}$, the Bragg wavelength diffracts out at $\theta_{\mathrm{B}}$ from the first grating and sets an angle of incidence $\theta_{\text {inc, } 2}=\theta_{\mathrm{B}}$ at the second grating that causes it to diffract again at $\theta_{\mathrm{B}}$ (see Fig. 8).

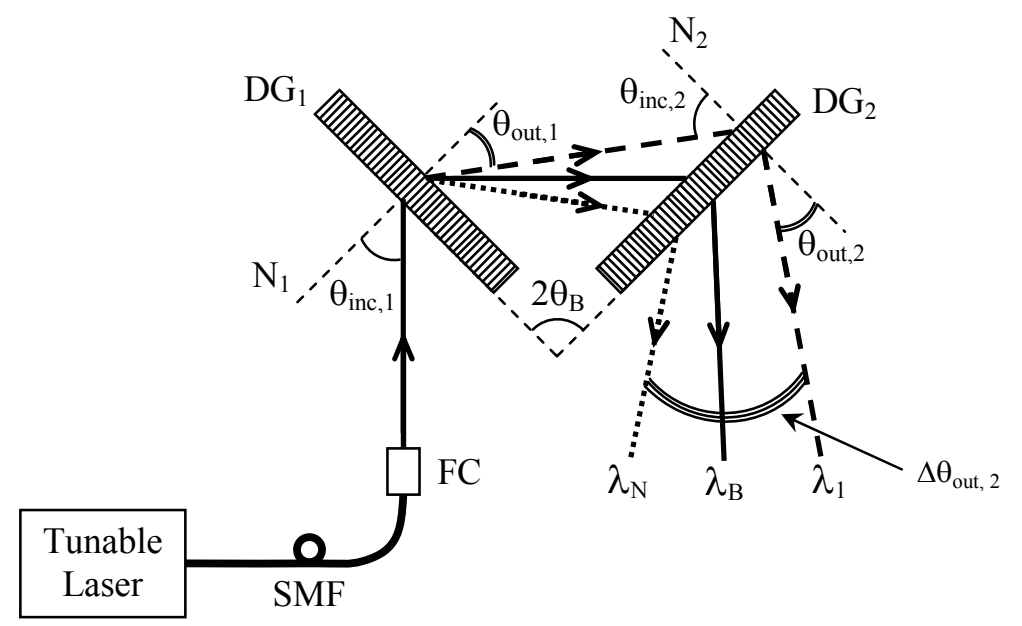

Figure 8: shows two similar Dickson gratings arranged in a symmetrical fashion (such that the angle between the planes of the two gratings is $2 \theta_{\mathrm{B}}$ ) for wide total angular scan range. $\mathrm{N}_{i}$ : Normal to the $i$ th grating; $\mathrm{DG}_{i}$ : $i$ th Dickson grating; SMF: Single mode fiber; FC: Fiber collimator (such as a gradient index lens).

As the wavelength of the laser source is tuned around the Bragg wavelength $\lambda_{\mathrm{B}}$, the angular scan after the first grating will be given by

$$
\theta_{\text {out }, 1}=\sin ^{-1}\left\{\frac{\lambda}{L}-\sin \theta_{\mathrm{B}}\right\} .
$$

The scanning beam from first Dickson grating will be incident on the second Dickson grating at an angle given by

$$
\theta_{\text {inc, } 2}=2 \theta_{\mathrm{B}}-\theta_{\text {out }, 1}
$$

where $\theta_{\text {out, }, 1}$ is given by Eq. (7). Hence, the final angular scan after the second Dickson grating (DG) will be

$$
\begin{aligned}
\theta_{\text {out }, 2} & =\sin ^{-1}\left\{\frac{\lambda}{L}-\sin \theta_{\text {inc, } 2}\right\} \\
& =\sin ^{-1}\left[\frac{\lambda}{L}-\sin \left\{2 \theta_{\mathrm{B}}-\sin ^{-1}\left(\frac{\lambda}{L}-\sin \theta_{\mathrm{B}}\right)\right\}\right] .
\end{aligned}
$$

Note that all the angles in Eqs. (7) to (9) are measured with respect to the grating normals $\mathrm{N}_{1}$ and $\mathrm{N}_{2}$. Thus the angular scan after $\mathrm{DG}_{1}$ and $\mathrm{DG}_{2}$ will be

$$
\Delta \theta_{\text {out }, 1}=\sin ^{-1}\left\{\frac{\lambda_{\mathrm{N}}}{L}-\sin \theta_{\mathrm{B}}\right\}-\sin ^{-1}\left\{\frac{\lambda_{1}}{L}-\sin \theta_{\mathrm{B}}\right\},
$$

and

$$
\Delta \theta_{\text {out }, 2}=\sin ^{-1}\left[\frac{\lambda_{\mathrm{N}}}{L}-\sin \left\{\sin ^{-1}\left(\frac{\lambda_{\mathrm{N}}}{L}-\sin \theta_{\mathrm{B}}\right)\right\}\right]-\sin ^{-1}\left[\frac{\lambda_{1}}{L}-\sin \left\{\sin ^{-1}\left(\frac{\lambda_{1}}{L}-\sin \theta_{\mathrm{B}}\right)\right\}\right],
$$

respectively. Finally, the setup in Fig. 8 is improved by adding two infrared mirrors for optical path folding to realize an in-line free-space W-MOS (see Fig. 9). Because the incident beam is horizontal, the Bragg wavelength $\lambda_{\mathrm{B}}$ diffracts out from $\mathrm{DG}_{1}$ at $\theta_{\mathrm{B}}$. The mirror $\mathrm{M}_{1}$ is oriented such that it makes an angle $-\theta_{\mathrm{B}}$ with the horizontal. The wavelength $\lambda_{\mathrm{B}}$ will 
therefore reflect from $M_{1}$ to become horizontal again. The mirror $M_{2}$ is vertically symmetric to $M_{1}$, i.e., it makes an angle $\theta_{B}$ with the horizontal which causes $\lambda_{B}$ to reach DG2 at $\theta_{\text {inc, } 2}=\theta_{B}$. Thus, the Bragg wavelength $\lambda_{B}$ diffracts from $\mathrm{DG}_{2}$ to come out horizontal. The analysis for angular deflection and total angular scan range as a function of wavelength (given above for the setup in Fig. 8) also holds for the one shown in Fig. 9, since both the setups are equivalent. Furthermore, Dickson gratings are transmissive volume Bragg gratings where the incident and diffracted beams are symmetric to the grating plane at Bragg wavelength. This indicates that a free-space W-MOS design using $n$ gratings $(n=1,2)$ will have a scanner aperture $d_{\text {out }}$ given by

$$
d_{\text {out }}=2 w_{\text {inc }} \cos \left(\theta_{\text {out }, n}-\theta_{\mathrm{B}}\right) \text {. }
$$

where $w_{\text {inc }}$ is the beam waist of incident Gaussian beam and $\theta_{\text {out }, n} ; \mathrm{n}=1,2$, is the output scan angle. It can be therefore concluded that although the scanner aperture will vary with the output scan angle, it will remain approximately the size of the incident beam since $\cos \left(\theta_{\text {out }, n}-\theta_{\mathrm{B}}\right)$ will be $\sim 1$.

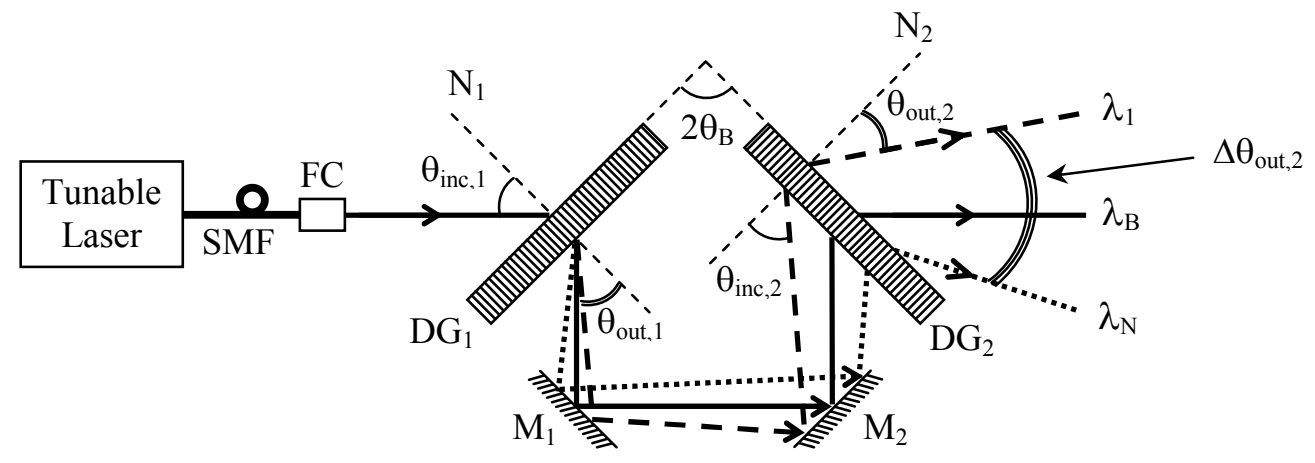

Figure 9: Schematic of an in-line wide angle free-space W-MOS using two Dickson gratings and two mirrors (for optical path folding). $\mathrm{N}_{i}$ : Normal to the $i$ th grating; $\mathrm{DG}_{i}$ : ith Dickson grating; $\mathrm{M}_{i}$ : $i$ th mirror; SMF: Single mode fiber; FC: Fiber collimator.

To study the angular scan available from the setup in Fig. 8 (or Fig. 9), a proof-of-concept experimental setup (shown in Fig. 10) is built in the laboratory. The experimental setup uses a Dickson grating and a mirror to simulate two Dickson gratings arranged symmetrically (in Fig. 8). Collimated beam from a fiber-coupled tunable laser (with $1 / \mathrm{e}^{2}$ beam size of $\sim 0.6 \mathrm{~mm}$ ) is incident on the grating mounted on a rotational stage to adjust the angle of incidence. The angle of incidence is set to $\theta_{\text {inc, } 1}=\theta_{\mathrm{B}}=46.76^{\circ}$ to satisfy the Bragg condition at $\lambda_{\mathrm{B}}=1550 \mathrm{~nm}$. An infrared mirror is placed close to the Dickson grating such that the diffracted beam at $\lambda=\lambda_{B}=1550 \mathrm{~nm}$ ray traces its own path, diffracts again and reaches the fiber collimator. An infrared camera is used to observe the scanning double diffracted beam as the wavelength of the tunable laser is tuned over the $80 \mathrm{~nm}$ wavelength band centered at $1560 \mathrm{~nm}$. The angular deflection is measured by tracking the scanning beam with the help of an iris mounted on an aluminum C-channel with one end attached to a rotational stage having the same axis of rotation as that for the Bragg grating.

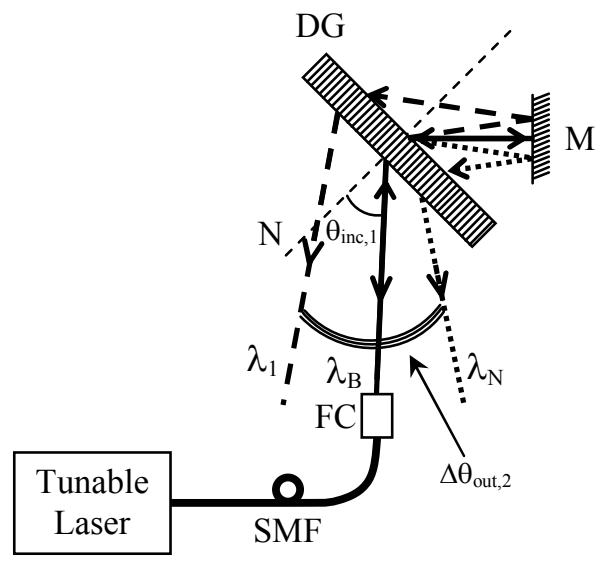

Figure 10: Experimental setup to simulate the in-line wide angle free-space W-MOS in Fig. 9 and study the angular scan range. 
A total angular scan of $13.42^{\circ}$ is measured using the setup in Fig. 10 as the wavelength of the source is changed from $1520 \mathrm{~nm}$ to $1600 \mathrm{~nm}$. It is important to notice that the measured scan is in complete agreement with the theoretical angular scan of $13.39^{\circ}$ estimated by Eq. (11) for the same $80 \mathrm{~nm}$ tunable bandwidth. Recall that the angular scan range available from the free-space W-MOS using a single Dickson grating is only $6.39^{\circ}$. It is therefore obvious that the angular scan range for an in-line W-MOS (using two Dickson gratings) is more than double the scan range that is available from the W-MOS design using a single Dickson grating. Figure 11 shows both experimental and theoretical angular scan (in degrees) versus wavelength of the tunable source.

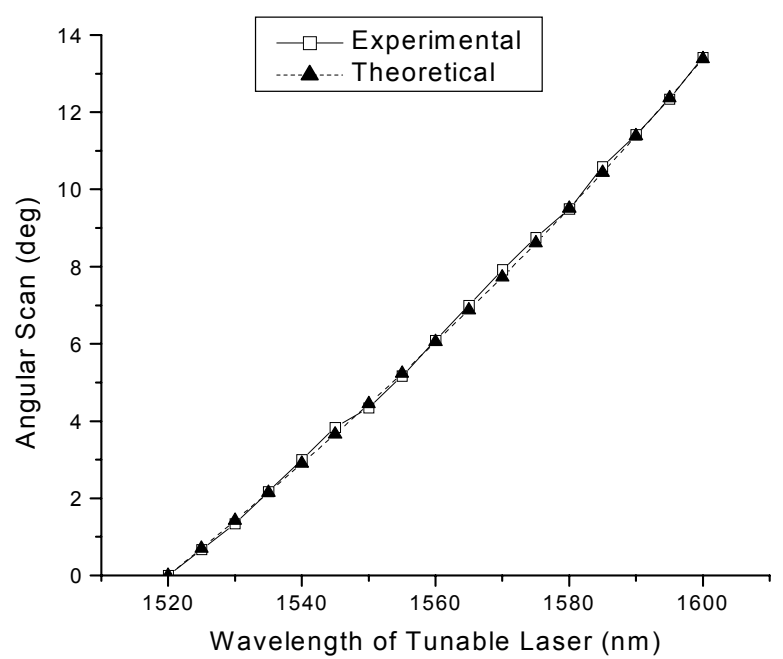

Figure 11: Free-space W-MOS angular scan (for two Dickson gratings) versus wavelength of the tunable source.

\section{CONCLUSION}

In conclusion, very high index of modulation is possible in dichromated gelatin that allows designing volume phase gratings (known as Dickson gratings) with very small thickness $(<10 \mu \mathrm{m})$, yet very high diffraction efficiency $(>95 \%)$. The small thickness of the dichromated gelatin layer in Dickson gratings makes them less selective in wavelength. The two characteristics, i.e., high diffraction efficiency and low wavelength selectivity of Dickson gratings make them suitable for use in the free-space W-MOS. Also, high spatial frequency $(940 \mathrm{lines} / \mathrm{mm})$ provides significant dispersion $\left(6.25^{\circ}\right.$ angular scan measured over $80 \mathrm{~nm}$ bandwidth around $\left.1560 \mathrm{~nm}\right)$. An in-line double scan range free-space W-MOS design for enhanced scanner operations is also discussed that uses two Dickson gratings and two mirrors for optical path folding. Experimental study (using a setup that simulates the dispersive capabilities of an in-line W-MOS) indicates that a total angular scan of $13.42^{\circ}$ is obtained when the laser source is tuned over $80 \mathrm{~nm}$ bandwidth around $1560 \mathrm{~nm}$. Furthermore, Dickson gratings are transmissive volume Bragg gratings where the incident and diffracted beams are symmetric to the grating plane at Bragg wavelength. This indicates that a free-space W-MOS design using such gratings will have a scanner aperture approximately the size of the incident beam. Using fast tunable lasers or optical filters coupled with a broadband source, the proposed free-space W-MOS features microsecond domain scan setting speeds, low scanner insertion loss, and single/multiple beam(s) in space. Because Dickson gratings are holographic phase gratings stored in dichromated gelatin layer sandwiched between glass plates, very large (e.g., several centimeters or more) diameter free-space W-MOS scanner apertures are possible for sub-degree angular scans. The potential speed of this scanner is in the Gigahertz range using present-day state-of-the-art nanosecond tuning speed lasers. ${ }^{15,16}$ Future work relates to the demonstration of the in-line free-space W-MOS with wide angular scan range capabilities.

\section{ACKNOWLEDGMENT}

This work is partially supported by DARPA grant N66001-98-D-6003. 


\section{REFERENCES}

1. P. J. Winzer and W. R. Leeb, "Space-borne optical communications - A challenging reality," Special Symposium on Agile Optical Beams and Applications, IEEE LEOS $15^{\text {th }}$ Annual Meeting, Invited Paper (WD2), Glasgow, Scotland, November 10-14, 2002.

2. G. S. Mecherle, "Active pointing for terrestrial free space optics," Special Symposium on Agile Optical Beams and Applications, IEEE LEOS $15^{\text {th }}$ Annual Meeting, Invited Paper (WL1), Glasgow, Scotland, November 10-14, 2002.

3. C. Chen, J. W. Alexender, H. Hemati, S. Monacos, T. Yan, S. Lee, J. R. Lesh, and S. Zingales, "System requirements for a deep space optical transceiver," in Free-Space Laser Communication Technologies XI, Proc. SPIE Vol. 3615, 142-152, San Jose, CA, 1999.

4. W. Klaus, "Development of LC optics for free-space laser communications," International Journal of Electronics and Communications, 56(4), 243-253 (2002).

5. R. L. Forward, "Passive beam-deflecting apparatus," US Patent No. 3,612,659, October 12, 1971.

6. K. G. Leib, "Radiation beam deflection system," US Patent No. 4,250,465, February 10, 1981.

7. Z. Yaqoob, A. A. Rizvi, and N. A. Riza, "Free-space wavelength-multiplexed optical scanner," Applied Optics-IP, 40(35), 6425-6438, December 10, 2001.

8. Z. Yaqoob and N. A. Riza, "Free-space wavelength-multiplexed optical scanner demonstration," Applied Optics-IP, 41(26), 5568-5573, September 10 (2002).

9. L. B. Glebov, N. V. Nikonorov, E. I. Panysheva, G. T. Petrovskii, V. V. Savvin, I. V. Tunimanova, and V. A. Tsekhomskii, "New ways to use photosensitive glasses for recording volume phase holograms," Opt. Spectrosc. 73, 237241 (1992).

10. O. M. Efimov, L. B. Glebov, L. N. Glebova, K. C. Richardson, and V. I. Smirnov, "High-efficiency Bragg gratings in photothermorefractive glass," Applied Optics-OT, 38(4), 619-627 (February 1999).

11. Z. Yaqoob, M. A. Arain, and N. A. Riza, "High-speed two-dimensional laser scanner by use of Bragg gratings in photothermorefractive glass," Applied Optics-IP, 42(26), September 10, 2003.

12. L. D. Dickson, "Method for making holographic optical elements with high diffraction efficiencies," US Patent No. 4,416,505, November 22, 1983.

13. H. Kogelnik, "Coupled wave theory for thick hologram gratings," Bell System Tech. J. 48, pp. 2909-2945, 1969.

14. A. Yariv and P. Yeh, Optical Waves in Crystals: Propagation and Control of Laser Radiation, John Wiley \& Sons, Inc. (1984).

15. G. Alibert, F. Delorme, P. Boulet, J. Landreau, H. Nakajima, "Subnanosecond tunable laser using a single electroabsorption tuning super structure grating," IEEE Photonics Technology Letters, 9(7), 895-897 (1997).

16. Delorme, G. Alibert, C. Ougier, S. Slempkes, and H. Nakajima, "Sampled-grating DBR lasers with 181 wavelengths over $44 \mathrm{~nm}$ and optimized power variation for WDM applications," in Optical Fiber Communication (OFC '98), 379-381 (1998). 